\title{
A propos des "Coches"
}

\author{
MICHEL CARLE
}

Je commence par celle qu'il me plaira, car les matières se tiennent toutes enchaînées les unes aux autres.

Essais, III, 5

Un chapitre qui n'est pas bien gros, un titre qui n'est pas bien clair, une foule de citations latines, une seule citation en grec et des idées. Voilà comment apparaît "Des coches," chapitre situé au centre du IIIe Livre des Essais. Placé entre "Sur des vers de Virgile," chapitre dans lequel Montaigne parle - entre autres - de l'écriture et de l'amour, et "De l'incommodité de la grandeur" qui parle de la grandeur et de ses incommodités, "Des coches" semble être posé là par hasard et n'avoir d'autre but que d'exacerber de temps à autre la perplexité des lecteurs de Montaigne. Pourtant, ainsi que nous allons essayer de le montrer, "Des coches" ne pouvait être ailleurs que dans ce Troisième Livre, dans lequel abondent les réflexions désabusées et parfois amères sur le pouvoir. A l'intérieur même du chapitre, on s'interroge sur une organisation, un agencement dans les thèmes abordés, on s'inquiète, en cherchant des repères, de ne pas "rater les 'Coches.'”

Le chapitre contient vingt-deux pages, ${ }^{1}$ trois pages sur les passions, huit pages sur les princes et leur politique, neuf pages sur le Nouveau Monde, le reste étant formé par les exemples que Montaigne utilise pour appuyer son argumentation. On s'interroge: quel lien peut-il exister entre des considérations sur les passions, des réflexions sur le pouvoir, des protestations contre les conquérants du Nouveau Monde? Et l'on s'étonne -déjà - que dans ce recensement n'apparaisse nulle part le sujet annoncé par le titre. Qu'est-ce à dire? Que Montaigne s'égare dès la première ligne? Que les coches sous-tendent malgré tout l'ensemble du chapitre, ou que l'auteur nous joue un bon tour? Il faudrait se demander si, paradoxalement, on ne pourrait pas répondre oui à ces trois questions. Montaigne s'égare; Montaigne se moque; Montaigne se sert des coches tout au long du chapitre. Il nous faut imaginer que l'auteur s'égare volontairement dans une foule de considérations dont la pertinence nous échappe et dont l'éventuelle unité ne peut surgir qu'en introduisant la perspective de l'ironie. Cette ironie fonctionnerait alors comme une sorte de garantie de la liberté d'expression, 
comme un voile atténuant la violence du réquisitoire contre le pouvoir auquel Montaigne se livre dans ce chapitre. Le titre même de celui-ci nous confirme cette dimension ironique, titre qui, par son caractère, laisse supposer un sujet tout autre que celui qui est traité. ${ }^{2}$ Les coches pourtant soustendent l'ensemble du chapitre, par la dimension presque symbolique dont Montaigne les revêt. Ils doivent être considérés comme les "véhicules" de la réflexion essentielle de Montaigne dans ce chapitre, réflexion portant sur les rapports entretenus par le pouvoir avec l'argent. Montaigne sait où il va, dans ce chapitre, tout en faisant semblant de s'égarer, pour son plus grand plaisir et pour la plus grande douleur de ses exégètes. Rappelonsnous Etiemble qui, passant en revue les différentes tentatives d'explication des "Coches," prétendait que le chapitre VI du Troisième Livre ne fonctionnait pas comme BOISDECAMPECHALALIGNE (sic). ${ }^{3}$ Cependant, Montaigne n'a-t-il pas songé au fait que l'on peut très bien parler de la colonisation et du pouvoir royal dans une dissertation sur les coches? Il nous faut admettre que ses considérations sur le pouvoir et ses protestations contre les conquistadores sont mûrement réfléchies, pesées et enveloppées dans l'innocent sujet des moyens de transport. Ne peut-on imaginer le malicieux Montaigne écrivant sur le mal au coeur et les coches et saisissant la balle au bond avec les coches de luxe qu'utilisaient les rois, en créant ainsi une transition anodine mais idoine? Anodine, parce que, ayant déjà parlé "des grands auteurs écrivant des causes" et s'étant retrouvé à parler des coches et du soulèvement d'estomac, il n'y a pas grand mal à parler maintenant des coches royaux et des rois; idoine, parce que la presque totalité du Troisième Livre baigne dans cette réflexion sur le pouvoir. ${ }^{4} \mathrm{Ce}$ qu'il y a à voir dans ces pages, c'est qu'elles sont situées au début du chapitre, c'est-à-dire dans la partie où Montaigne cherche, en quelque sorte, la bonne tonalité. Ces pages ne contiennent pas la première de trois grandes idées, ${ }^{5}$ elles sont situées avant deux grandes idées; ou plus exactement avant les deux volets d'un même commentaire sur l'argent et la politique de la société européenne.

C'est par un trait de coquette que Montaigne aborde la première grande idée du chapitre, les princes et la politique. Lorsque Montaigne nous dit:

Si j’avais la mémoire suffisamment informée, je ne plaindrais mon temps à dire l'infinie variété que les histoires nous présentent de l'usage des coches, (p. 148)

ne le croyons pas. Il y a là un procédé de fin écrivain, de "coquette," qui prétend ne rien savoir d'un sujet qu'il traite malgré tout sur une page. Il se moque un peu, à la fin du paragraphe, de ceux qui l'ont cru et qui déjà déploraient sa mauvaise mémoire, lorsqu'il déclare: "mais laissons ces 
coches guerriers." Eh! Quoi! Montaigne est seul à parler, (c'est à la première personne du singulier que débute le paragraphe) et voilà que par caprice il associe le lecteur à ses divagations! Croyons bien plutôt que Montaigne cesse de vérifier si sa mémoire est bien informée. Il se moque. S’il s'égare. c'est volontairement et l'exemple que nous venons de voir se reproduira à la fin du chapitre avec le fameux "retombons à nos coches." Ces réflexions abruptes, jetées au milieu du texte de loin en loin, agissent comme autant de gigantesques parenthèses refermées. Sans diminuer l'importance des mots qu'elles contiennent, ces parenthèses permettent à l'auteur d'atténuer un peu des propos sentant soit le fagot, soit la paille humide. C'est ce qu'il nous faudra avoir à l'esprit lorsque nous aborderons le problème de la colonisation dans "Des coches." Nous avons là, à une échelle moyenne, (le chapitre se situe, quant à la taille, à mi-chemin entre le paragraphe et le livre) un exemple du style de Montaigne. Sa garantie, c'est le fil de la plume qu'il prétend suivre; ainsi peut-on croire qu'il s'égare et c'est ce qu'il souhaite. Faisant semblant de rien, il suit doucement son idée (qu'il embrouille volontairement dans le cul-de-sac du "mais laissons ces coches guerriers"). Mais le fil qu'il suit n'est pas celui de sa plume. C'est un fil qu 'il sait solidement noué à l'extrémité de son chapitre. Tout en le suivant, Montaigne visite les pages par où passe ce fil, c'est-à-dire des pages de conseils et de condamnation. C'est un seul et même fil qui traverse tout le chapitre, lâchement tendu, de section irrégulière et dont l'irrégularité même sert de point de repère. La grosseur du fil est identique lorsqu'il est question du Pont Neuf et de la route de Quito à Cusco, ${ }^{6}$ identique aussi entre l'empereur Probus et "l'espovantable magnificence des villes de Cusco et de Mexico."

Nous commençons à y voir plus clair: un chapitre ("Des coches"), une idée (le pouvoir), deux volets (la politique des princes et la colonisation du Nouveau Monde). Nous pouvons considérer que le passage sur la politique des princes commence avec "Marc Antoine fut le premier qui se fit mener à Rome," ${ }^{8}$ et s'achève huit pages plus loin pour laisser une place (déjà aménagée) à la seconde partie du commentaire. Dans ce passage, Montaigne cite trois noms de dirigeants romains qui utilisèrent des coches pompeusement parés.

Les exemples qu'il nous donne sont choisis évidemment pour servir au but visé. Trois hommes séparés par quatre cents ans d'histoire eurent les mêmes désirs de faste, cela n'échappe pas à Montaigne. ${ }^{9}$ Une telle constance dans cette espèce de "pusillanimité aux monarques" va lui permettre de tirer une règle générale qu'il va appliquer aux princes de son époque. Certes, cela sera fait tout en douceur; Montaigne va multiplier les exemples historiques, citer Isocrate, Démosthène, Théophraste, Aristote, flatter Grégoire XIII et Catherine de Médicis, parler de lui-même quand il était cadet; mais toute cette poudre, une fois soufflée, laisse apparaître un paragraphe fort court, aussi tranchant qu'une maxime: 
La juridiction ne se domine point en faveur du juridiciant, c'est en faveur du juridicié. On fait un supérieur, non jamais pour son profit, ainsi pour le profit de l'inférieur, et un médecin pour le malade, non pour soi. Toute magistrature, comme tout art, jette sa fin hors d'elle. Nulla ars in se versatur. (p. 151).

Remarquons comment Montaigne fait alterner le passé et le présent et comment, par vagues successives d'abord infimes puis de plus en plus fortes, jaillit la sentence. Trois noms de dirigeants romains, plus celui d'Isocrate: passé. "J'aimais à me parer"; Montaigne, XVIe siècle: présent. Démosthène, Aristote: passé. Grégoire XIII, Catherine de Médicis: présent. Galba: passé. C'est-à-dire que la proportion passé-présent alterne tout en marquant la diminution de l'un et l'accroissement de l'autre:

$\begin{array}{lccccc}\text { Epoque: } & \text { passé } & \text { présent } & \text { passé } & \text { présent } & \text { passé } \\ \text { Nombre de personnages: } & \text { IV } & 1 & \text { II } & 2 & \text { I }\end{array}$

Relevons à présent le "Par quoi" qui ouvre le paragraphe suivant et qui est un indice montrant que nous sommes en présence d'une démonstration rigoureuse. Nous trouvons d'autres indices plus haut, cachés dans les anecdotes: "car,"10 "outre ce,"11 sont les instruments rhétoriques d'un implacable raisonnement. Ils amènent Montaigne à formuler fermement, sans crainte, l'idée selon laquelle le roi "se doit soi-même à autrui." C'est sur le même ton qu'il dénonce "les gouverneurs de l'enfance des princes" qui "regardent plus à leur profit qu'à celui de leur maître." Insensiblement Montaigne, dans ces pages, élabore une théorie du pouvoir assez semblable à celle exprimée dans Le Prince. ${ }^{12} \mathrm{Ce}$ rapprochement ne nous surprend plus depuis longtemps. ${ }^{13}$ Mais il nous faut malgré tout évoquer Machiavel lorqu'on lit "Des coches," parce que ce chapitre est composé de conseils et de mises en garde adressés aux dirigeants, parce que, en le rédigeant, Montaigne s'inquiète de la popularité des princes, tout comme dans Le Prince Machiavel s'acharne à expliquer l'importance pour le prince, de son renom dans l'opinion publique. ${ }^{14} \mathrm{Du}$ Prince, le chapitre XVI notamment, "De la générosité et de la parcimonie," doit attirer notre attention par la parenté de vues que l'on y découvre avec les réflexions de Montaigne sur la "libéralité" ou la "frugalité" du prince; lorsque Machiavel conseille au prince de "ne pas craindre le nom de ladre" alors que Montaigne déclare qu'il "aime mieux avare" un prince dont la libéralité est "sans discrétion," nous sommes obligés d'admettre que le discours de Montaigne sur le pouvoir, à la forme près, serre de très près le discours politique du théoricien florentin; et d'admettre, en conséquence de quoi, que "Des coches," sous l'apparence d'un chaos inextricable, est "un véritable traité en forme d'avertissement aux finances royales," selon la formule de 
Géralde Nakam. ${ }^{15}$ D’ailleurs cette rigueur rhétorique des "Coches" est particulièrement sensible lorsque Montaigne explique la manière dont il faudrait dépenser l'argent: des ports, des havres, des fortifications, des murs, des églises, des hôpitaux, des collèges, des rues et des chemins; c'està-dire employer l'argent d'une façon "utile, juste et durable." Parallèlement à l'idéal humaniste qui perce sous ces lignes, l'énumération des points sur lesquels devrait agir la libéralité des rois - énumération d'une belle qualité rhétorique - annonce les pages vibrantes sur le Nouveau Monde. Par la qualité de l'expression, nous sommes sûrs d'être en présence de la grande idée de Montaigne dans ce chapitre. Les phrases, d'une facture parfaite, sont aussi rigoureuses que des articles de loi:

... Les sujets d'un prince excessif en don se rendent excessifs en demande ....... La vertu royale semble consister le plus en la justice ....;. . Nous sommes surpayés selon justice quand la récompense égale à notre service ....... Si (le prince) porte notre dépense, il fait trop ...;

elles tirent leur force de leur construction rigide où chaque mot rend un son plein. Ailleurs, ne nous laissons-nous pas entraîner à rêver lorsque Montaigne nous conte les folies de l'empereur Probus? L'anecdote qu'il nous livre commence comme un conte de fées. Et Montaigne en est parfaitement conscient lorsqu'il multiplie les descriptions dans des phrases longues, entrecoupées de virgules qui rendent le texte haletant. La description des arbres, l'énumération des animaux sauvages, les chiffres "merveilleux" qu'il donne (mille autruches, mille cerfs, mille sangliers, mille daims, cent gros lions, cent léopards, trois cents ours, trois cents paires de gladiateurs (p. 154), tout concourt à faire rêver le lecteur. Il y a lieu d'admirer dans ces deux pages l'art de Montaigne qui cisèle et qui émaille de citations latines un texte déjà riche en descriptions fastueuses. Mais, dans la première phrase, ("C'était pourtant ${ }^{16}$ une belle chose. .."), ne trouve-t-on pas un rien de nostalgie? Si ces "jeux et montres publiques" ne sont que "superfluité," pourquoi donc en parler pendant deux pages? N'y a-t-il pas lieu de voir ici une épitrope, une figure de style qui, nous dit Fontanier, "dans la vue même de nous détourner d'un excès (. . .) semble nous inviter à nous y livrer?"17 Il en sera de même lorsque Montaigne parlera avec admiration de "l'épouvantable magnificence" des villes du Nouveau Monde. Bien que ces deux pages ne soient que des pages d'exemples, mises entre parenthèses par la répétition du mot "excuse," 18 leur chaleur nous invite à penser que Montaigne n'était pas aussi insensible qu'il le dit à de "tels excès." Qu'il prenne parti en faveur de l'économie des finances du royaume, qu'il conseille aux princes de "semer de la main, non pas verser du sac," les exemples que donne Montaigne semblent trahir son intention. Montaigne est fasciné par le luxe, l'opulence, les richesses du pouvoir. Aussi n'a-t-il 
pas hésité entre une place dans le gouvernement de Henri IV (qui lui aurait permis d'approcher le faste du pouvoir) et sa tranquillité. L'attitude de Montaigne, en cette occasion encore, illustre bien son goût du confort; s'il aime le luxe que dispense le pouvoir, c'est celui de l'Antiquité, celui décrit par Calpurnius et Martial, une richesse inouïe et dont les froides salles du Louvre ne devaient être qu'un pâle reflet. Montaigne suit les évolutions de la cour, mais de loin, dans son château, dans sa "librairie." Le luxe et la richesse des princes, il préfère les voir dans un de leurs pays de naissance, l'Italie, où il va séjourner longuement. ${ }^{19}$ Sous cet aspect, il faudrait peutêtre se demander si sa condamnation des agissements des Espagnols au Nouveau Monde ne lui est pas dictée en partie par une révolte contre le pillage de la richesse, contre la destruction du luxe. Le ton de la description de l'"épouvantable magnificence des villes de Cusco et de Mexico" ne va pas sans ramener en nous l'écho de sa description des jeux de Probus. Voilà un lien nouveau entre les deux grandes parties du chapitre, pour nous qui nous demandions si Montaigne s'égarait. Les coches de luxe ont été un prétexte à parler du luxe, de la richesse et, par une démarche fort logique, à parler de l'endroit où se trouve cette richesse, à parler de son propriétaire, du pouvoir. La transition est faite par Montaigne lui-même qui met "les points sur les i pour qui n'aurait pas compris l'unité des 'Coches," (selon la formule d'Etiemble). Le parallèle est constant entre le passé et le présent et le "nous nous promenons sur nos pas" a la même force expressive que la "branloire pérenne" du chapitre "Du repentir." Que la formule des "Coches" fasse allusion à la narration de Solon - et en ce cas le "sur nos pas" est sans doute pris au sens propre ${ }^{20}$ - ou que de façon plus générale elle renvoie à une intention pédagogique, elle indique clairement l'existence d'un plan préétabli dans l'esprit de son auteur.

N'y-a-t-il pas lieu de voir dans l'apparition des noms d'Egypte et de Chine la volonté de Montaigne d'ouvrir progressivement le champ de ses commentaires et d'amener ainsi, par deux allusions à des pays étrangers, des propos sur le Nouveau Monde? Il nous faut nous souvenir que l'évocation de la Chine avait dans les mentalités de l'époque une résonance exotique aussi forte que l'évocation du Nouveau Monde. Ces deux noms ont eu, à quelques années d'intervalle, la même force, même si la découverte de Christophe Colomb fit oublier les récits de Marco Polo. ${ }^{21}$ La Chine, dans les quelques lignes que Montaigne lui consacre, représente "un membre perclus," un univers tombé "en paralysie," qui conçut "mille ans auparavant" la poudre et l'imprimerie. Aux parallèles temporels se surajoute un parallèle spatial. Quelle sagesse, quelle humilité et quelle grandeur (et tant pis pour l'emphase) dans cette candide constatation: "Notre monde vient d'en trouver un autre"! Cette phrase ouvre une longue plaidoirie sur la notion de civilisation. C'est par le biais de la condamnation des Espagnols que Montaigne condamne la société européenne. Le premier paragraphe 
(p. 158) termine la transition amorcée deux pages plus haut et tire les conclusions de cette espèce d'humilité sceptique sur la notion de savoir. La question: "et qui nous répond si c'est le dernier de ses frères?" est la conséquence logique des réflexions sur la connaissance "combien chétive et raccourcie ... . des plus curieux."

C'est par le biais de la condamnation des Espagnols que Montaigne condamne la société eu ropéenne, en présentant les implications qu'eut sur le Nouveau Monde le manque d'humilité de l'Europe. Remplis d'un orgueil non fondé, les conquistadores établirent des rapports de supérieurs à inférieurs avec les Indiens. Lorsqu'on s'appelle Michel de Montaigne et qu'on a écrit 1"'Apologie de Raymond Sebon", on ne peut laisser passer une telle outrecuidance. Celui qui avait écrit:

C'est le déjeuner d'un petit ver que le coeur et la vie d'un grand et puissant empereur (Essais, II, 12.)

pouvait difficilement supporter l'idée d'une quelconque supériorité d'un homme sur un autre. Lorsque Montaigne, page après page, nous a démontré qu'en fait il n'y a pas de quoi se croire supérieur aux animaux, ${ }^{22}$ comment pourrait-il se taire quand nous opprimons un autre peuple? Montaigne a démontré, dans 1"“Apologie," le caractère relatif de cette notion de supériorité; il la détruit dans "Des coches" en présentant les moeurs et les coutumes, et surtout l'existence de la raison chez les Indiens. Dans un temps où ce mot est l'élément premier en matière de comparaison. Montaigne déclare que les habitants du Nouveau Monde "ne nous devaient rien en clarté d'esprit naturelle et pertinence." Il en va de même pour leurs productions dans le domaine de l'esthétique; "ils ne nous cèdent non plus." Le paragraphe suivant (p. 159), qui rappelle tant les pages de 1"“Apologie," (quant à la présentation de l'argumentation), illustre la vision planétaire de Montaigne. ${ }^{23}$ Ceci nous amène à nous demander s'il fut le seul de son temps à défendre les Indiens. En rappelant Bodin et Gomora, il faut aussi nous souvenir de Bartholomé de Las Casas, le seul qui consacra plusieurs ouvrages à la situation des Indiens d'Amérique. ${ }^{24}$

Mais la position de Las Casas est différente de celle de Montaigne. Montaigne fut le seul à prendre la défense des habitants du Nouveau Monde par amour de l'humanité. En lisant Las Casas, on se rend compte qu'il pose le problème dans une perspective politique et religieuse. D'autre part, il faut tenir compte du fait que Montaigne s'est insurgé contre le sort des Indiens sans être directement concerné, comme c'était le cas de l'évêque de Chiapas. ${ }^{25}$ Ceci n'enlève rien à la gloire de Las Casas mais grandit celle de Montaigne. Il importe peu ici de poser la question "pourquoi et comment Montaigne s'intéressa-t-il au Nouveau Monde?" Il était humaniste, que cela nous suffise. Les ouvrages sur le Nouveau Monde (Floride, France 
antarctique, etc.) ne manquaient pas en France à l'époque où Montaigne écrivait ses Essais. ${ }^{26}$ Retenons que Montaigne s'intéressa sérieusement au Nouveau Monde, se renseigna le mieux qu'il put, chercha l'objectivité dans les relations orales qu'on lui fit (on se souvient des précautions qu'il prend surle quotient intellectuel de son témoin). Le principal reproche sur lequel va se fonder l'accusation de Montaigne est relatif à la cupidité des Espagnols. L'argument religieux que ceux-ci brandissaient, Montaigne le repousse avec fin de non recevoir:

Serait-ce zèle envers la religion? Ce sont voies trop diverses et ennemies d'une si sainte fin. (p. 164)

Montaigne dénie aux Espagnols le droit de s'abriter derrière le prétexte religieux pour expliquer leur cruauté. La seule et unique raison de la conquête est contenue tout entière dans la très belle phrase:

Tant de villes rasées, tant de nations exterminées, tant de millions de peuples passés au fil de l'épée, et la plus riche et belle partie du monde bouleversée pour la négociation des perles et du poivre! (p. 161)

L'or, les perles et le poivre, voilà le vrai motif de la conquête, une action économique dont Montaigne dresse le bilan:

... la recette ... répond ... peu à l'espérance qu'on en donna.

L'unité des "Coches," c'est bien l'argent. Dans ce même paragraphe (p. 165), nous trouvons l'écho du vers de Corinne: "Nous l'épandons (l'or) et dispersons." Montaigne eût préféré que cet or volé au Nouveau Monde fût conservé par les rois d'Europe plutôt que d'être "altéré en mille formes." Voilà qui est un paradoxe pour quelqu'un qui souhaitait, quelques pages plus haut, que l'or des princes servit dans de royaux emplois. En fait nous voyons confirmé ici le goût de Montaigne - goût tout esthétique - pour la richesse. Revenons une fois encore sur la description des jardins de ce roi du Nouveau Monde,

Où tous les arbres, les fruits et toutes les herbes, selon l'ordre et la grandeur qu'ils ont en un jardin étaient excellemment formés en or. (p. 159)

Il en est de même pour "tous les animaux qui naissaient en son Etat ou en ses mers." Si on relève les chiffres de la rançon d'Attabalipa et les chiffres des victimes qui périrent "pour un coup, en même feu" qu'il donne, on se rend compte que c'est le gros et le surabondant qui impressionne Montaigne. Ainsi précise-t-il que les chevaux des Espagnols "n’allaient plus ferrés que d'or massif." 
Ceci ne signifie pas que l'on doive mettre en doute la valeur de son indignation. Sa prise de position et sa colère contre les Espagnols sont tout à fait dignes de foi. On le constate avec le changement de ton entre "Des Cannibales" et "Des coches." C'est parce qu'il s'intéresse à la nouveauté que Montaigne essaie d'expliquer les moeurs des Brésiliens, et c'est parce qu'il est choqué par la violence qu'il dénonce la cruauté des Espagnols. S'il plaisante un peu sur les Cannibales, s'il est relativement dégagé de son sujet, il est au contraire pleinement "engagé" dans "Des coches." ${ }^{27}$ Le ton de toute la seconde partie du chapitre nous fait comprendre que le fameux sourire de Montaigne se transforme en grincement de dents. La longueur des phrases, les chiffres, les anecdotes qu'il nous livre sont loin, bien loin du

Tout cela ne va pas trop mal: mais quoi ils ne portent point de hauts-dechausses! ${ }^{28}$

Il faudrait enfin se demander si la colère de Montaigne n'était pas également liée en partie au massacre des Français de Floride, à savoir si Montaigne défend les Indiens ou condamne les Espagnols. Les ouvrages traitant de cet épisode de la colonisation avaient paru en français entre 1566 et $1568 ; 29$ Montaigne aurait pu les connaître. Dans cette hypothèse, il faudrait voir dans son attitude une forme de patriotisme, qui n'a rien d'incompatible avec son humanisme. On peut aimer son pays et "se prêter" à de nobles causes étrangères. C'est bien parce qu'il dit

Jestime tous les hommes mes compatriotes, non parce que Socrate l'a dit mais parce que c'est mon humeur (Essais III, 9)

qu'on peut l'imaginer sujet du roi de France et citoyen du monde.

Notons une dernière fois l'unité qui règne dans "Des coches." Par ce même mouvement de va et vient qui rappelle au présent qu'il rejoue les scènes du passé, Montaigne referme son chapitre:

Quant à la pompe et la magnificence, par où je suis rentré en ce propos, ni Grèce, ni Rome, ni Egypte, ne peut comparer aucun de ses ouvrages au chemin qui se voit au Pérou. (p. 166)

Dans cet avant-dernier paragraphe, Montaigne fait la synthèse de tout ce qu'il a dit au-dessus. Il a parlé de l'Antiquité grecque et latine, de l'Egyptepar son allusion au Timée - et du Pont Neuf inachevé. Il nous montre clairement ce qu'il faut retenir de son chapitre et surtout ce qu'il faut y lire. Il ne nous reste plus qu'à retomber à nos coches, ce qui se fait fort naturellement, ayant parlé de routes peu avant. Cette formule pourrait rappeler la dernière phrase des "Cannibales". Le ton pourtant est bien différent. A la 


\section{pirouette finale des "Cannibales", Montaigne préfère ici porter un dernier} coup à la cruauté espagnole. Aussitôt que l'on retrouve le sujet du titre, on repart au Pérou voir l'Inca tomber sous les coups de Pizarre. ${ }^{30}$ Et c'est sur cette image que s'achève "Des coches", sur une vision d'or, de sang et de destin.

\section{Université d'Ottawa}

\section{Notes}

1 Dans l'édition du Livre de Poche, présentée par P. Michel, (Paris, 1972). Cette édition a été faite sur l'exemplaire de Bordeaux; c'est à elle que nous renvoyons. Afin d'alléger les notes, seul apparaîtrae le numéro de la page et il sera donné à la suite de la citation. Qu il me soit permis ici de remercier M. Michael Liddle, à qui doit tant la version finale de cet article.

2 Pierre Villey avait signalé "lironie continuelle" des "Coches" et la justifiait par une succession de développements "composés au fur et à mesure des lectures de Montaigne". En voyant dans ce chapitre la matière de trois chapitres distincts, P. Villey notait: "Ce titre est là pour surprendre". Les sources et l'évolution des Essais de Montaigne, 2 vol. (Paris, Hachette, 1908) II, 287.

3 Etiemble, “C"est le bouquet!", Hygiène des lettres, t. V,(Paris, Gallimard, 1967) pp. 101-115. Pour une liste complète des tentatives d'explication des "Coches," nous renvoyons à l'article de Marcel Gutwirth: "'Des coches,' ou la structuration d'une absence," in L'Esprit créateur, University of Kansas, 15 (1975) 8-20.

4 Voir notamment Géralde Nakam, Les Essais de Montaigne, miroir et procès de leur temps (Paris: Nizet, 1984), pp. 152 et sq.

5 Que P.Villey résume ainsi: un premier développement sur les malaises quéprouve Montaigne "en coche, en litière aussi bien qu'en bateau," et "un excursus sur la passion de la peur", un second qui "traite de la libéralité chez les rois", un troisième "sur les cruautés des Espagnols aux Indes". P. Villey, op. cit., II, 286.

6 pp. $150 ; 166$.

7 pp. $154 ; 159$.

8 p. 149.

9 Marc Antoine meurt en 30 av. J.-C., Frimus en 372 ap. J.-C.; N.B. Montaigne choisit trois personnages qui mirent volontairement fin à leurs jours.

10 p. 150; à deux endroits, dernier paragraphe.

11 p. 150; au début du dernier paragraphe.

12 Publié un an avant la naissance de Montaigne.

13 Après Joseph Barrère et Pierre Michel, c'est Alexandre Nicolaï qui a repris et développé l'idée du "machiavélisme de Montaigne" dans une série d'articles, Bulletin de la Société des Amis de Montaigne, 4,7,9(1957-1959), où il déclarait que certaines pages des Essais étaient à lire comme une "Institution du Prince," "toutes entières inprégnées de la pensée de Machiavel" (Ibid., No 7,p. 3). En 1966, Anna Maria Battista, dans Alle origini del penserio politico libertino: Montaignee Charron (Milan, 1966); en 1972, Machiavel et Montaigne, est le sujet d'une thèse de troisième cycle, rédigée par Pierre Goumarre; voir également Géralde Nakam, Les Essais de Montaigne, miroir et procès de leur temps, Première partie, chap. VII, "Montaigne, Bodin et Machiavel" (Paris, Nizet, 1984), p. 244 et sq.

14 C'est ce que signale Géralde Nakam (op. cit. p. 249) en rappelant que "le renom du prince est chose essentielle; Machiavel y consacre près du tiers de son livre."

15 G. Nakam, op. cit. p. 58.

$16 C^{\prime}$ 'est nous qui soulignons.

17 P. Fontanier, Les figures du discours, (rééd. Flammarion: Paris, 1977), p. 148. 
18 p. 153: "Les empereurs tiraient excuse ..."; p. 156: "S"il y a quelque chose qui soit excusable...."

19 Il va rester cinq mois à Rome.

20 Ceci peut être lu comme une allusion directe à la "découverte" du Nouveau Monde, par la référence qui est faite au Timée dans lequel Solon parle de l'Atlantide. C'est l'Atlantide que de nombreux esprits du XVIe siècle crurent/voulurent reconnaître dans l'Amérique.

21 Cf. G. Chinard, L'exotisme américain dans la littérature française au XVle siècle (Paris: Hachette, 1911).

22 Dans 1".Apologie..."

23 Il est intéressant en effet de constater que la description qu'il va faire de l'équipement des Espagnols passera par l'utilisation d'un vocabulaire imagé, d'une "couleur locale," sil'on veut, prêté aux Indiens. Les grands monstres inconnus, les armes tranchantes et resplendissantes, la peau luisante et dure, pour cheval, épée, cuirasse, sont un raccourci habile pour faire comprendre l'état d'esprit des Indiens et le peu de mérite des Espagnols.

24 B. de Las Casas, Histoire admirable des horribles insolences, cruautez et tyrannies exercées par les Espagnols ès Indes occidentales . . fidèlement traduite par Jacques de Miggrode, s.1., 1582. - Tyrannies et cruautez des Espagnols perpétrées éès Indes occidentales ... traduit par Jacques de Miggrode, Paris, 1579. (Il y eut plusieurs éditions). Les dates nous permettent d'imaginer que Montaigne en prit connaissance. P. Villey affirme que "Des coches" n`a pu être composé avant 1584. Marcel Bataillon, dans "Montaigne et les conquérants de l’or," Les langues néolatines, 1973, No 204, pp. 31-50, avance que Montaigne n’avait pas lu Las Casas, ce qui paraît "impossible" à G. Nakam (op. cit. p. 340).

25 Nous n'avons pas ici à entrer dans les détails de l'attitude de Las Casas. Une bonne mise au point a été faite par Edmundo O'Gorman, dans son édition de Los indios de México y nueva España (Mexico: editorial Porrúa, 1974), 3ème édition.

26 Cf. G. Atkinson La littérature géographique française de la Renaissance, Répertoire bibliographique, (Paris: A. Picard, 1927).

27 Géralde Nakam note très pertinemment l'écart de huit ans existant entre l'édition des "Cannibales" et celle des "Coches": "il suffit de comparer les pages de 1580 consacrées aux Indiens à celles de 1588. Dans les premières, le monde indien vit encore. Dans les secondes, il n'est plus." (op. cit. p. 311) La fin d'un monde dont nous avons vu la fascination que son opulence exerçait sur Montaigne peut bien être la cause de la colère de l'auteur des "Coches".

28 Dernière phrase des "Cannibales", Essais, I, 32.

29 On relève dans G. Atkinson, op. cit, quatre ouvrages sur ce massacre. Deux de N. Le Challeux, accompagnés d'une Requeste présentée au Roy par les femmes veufves et enfans orphelins, parens et amis de ses sujets, (tous deux de 1566); un anonyme sur l'Histoire mémorable de la reprinse del'isle de la Floride, en 1568; une Petite histoire d'un massacre commis par les Hespagnols sur quelques Français en la Floride, en 1579.

30 N'est-ce pas par mépris que Montaigne ne définit pas "l'homme de cheval" comme étant F. Pizarre? 
